\title{
User requirements for prediabetes self-care application: a healthcare professional perspective
}

\begin{abstract}
The number of people with diabetes is increasing dreadfully in the world today. Efforts to prevent diabetes should be taken at the prediabetes stage itself, because with proper lifestyle changes, diabetes can be prevented or delayed. As such, a self-care application can help prediabetics to monitor their own health to prevent diabetes. The objective of this study is to explore the healthcare professionalsôperspectives that contribute to behavioural change in prediabetics and the user requirements for a self-care application. We conducted semistructured interviews with eight healthcare professionals. Pre-determined set of open-ended questions were asked by the interviewer to prompt the discussion. Thematic Analysis approach was used to evaluate the responses. Overall, the health professionals were keen with the idea of empowering prediabetics to take control of their health conditions via a self-care application incorporating behaviour change techniques. Several functions were suggested to be included into the application such as forums to allow discussions among the prediabetics, coaching to communicate with healthcare professionals and peer/family support to motivate them to use the applications continuously.
\end{abstract}

Keyword: Self-care; Behavioural change; Blood glucose; Diabetes prevention; Mobile application; Web technology 